\title{
An Improved Bernsen Algorithm Approaches For License Plate Recognition
}

\author{
Madhuri Latha.G ${ }^{1}$, Chakravarthy. $G^{2}$ \\ ${ }^{1,2}$ (ECE, Velagapudi Ramakrishna Siddhartha Engineering College, Vijayawada)
}

\begin{abstract}
An Improved Bernsen Algorithm approaches for License Plate Recognition (LPR) applied to the intelligent transportation system. LPR is an image processing technology used to identify the vehicles by their license plates. License plate recognition is mainly based on two methods. One is License plate detection method which is based on Improved Bernsen Algorithm and the other is license plate character recognition method. The proposed LPR algorithm consists of three modules: 1) Locating the license plates; 2) Segmenting the characters; and 3) Identifying the license characters. The algorithm was tested with 100 images, such as an Indian vehicle images using different backgrounds. The experimental results show the algorithm can divide the character of plate effectively.
\end{abstract}

Keywords: Bernsen algorithm, character recognition, feature extraction, License Plate Recognition.

\section{INTRODUCTION}

The problem faced by the motorists using toll roads is the congestion and delay caused by stopping to pay at the tollbooths. The solution for this problem is Electronic Toll Collection (ETC). ETC is generally broken up into three parts. Automatic Vehicle Classification (AVC), Automatic Vehicle Identification (AVI) and Violation Enforcement System (VES). License plate detection, while rarely used as the primary vehicle identification method and commonly used in the violation enforcement.

Various license plate detection algorithms have been developed in past few years. Each of these algorithms has their own advantages and disadvantages. One of the method in which license plate is detected using confidence related predictions. As multiple detections are available for single license plate, post processing methods are applied to merge all detected regions. In addition, trackers are used to limit the search region to certain areas in an image. Another approach for detection is binarization[2] and elimination of unnecessary regions from an image. In this approach, initial image processing and binarization of an image is carried out based on the contrast between characters and background in license plate. After binarizing the image, it is divided into different black and white regions. These regions are passed through elimination stage to get the final region[4] having most probability of containing a number plate.

Researchers have proposed many methods of locating the license plates, such as the edge detection method line sensitive filters to extract the plate areas, the window method, and the mathematics morphology method[3]. Although these algorithms can process the license plates location, they possess formidable disadvantages such as sensitivity to brightness [5], longer processing time, and lack of versatility[7] in adapting to the varying environment.

The main purpose of this project is to detect a license plate from an image provided by a camera. Work focus on a solution for image disturbance resulting for uneven illumination and various outdoor conditions such as shadow and exposure while generally difficult for obtaining successful processed results using traditional methods[10]. An efficient algorithm is developed to detect a license plate in various luminance conditions. Improved Bernsen Algorithm extracts the license plate data from an image and provides it as an input to the stage of Car License Plate Recognition. The image of a vehicle is given as an input from the camera. Extracted image of the number plate can be seen on monitor for verification purpose.

\section{LICENSE PLATE DETECTION ALGORITHM}

The flowchart of license plate detection algorithm as shown below 


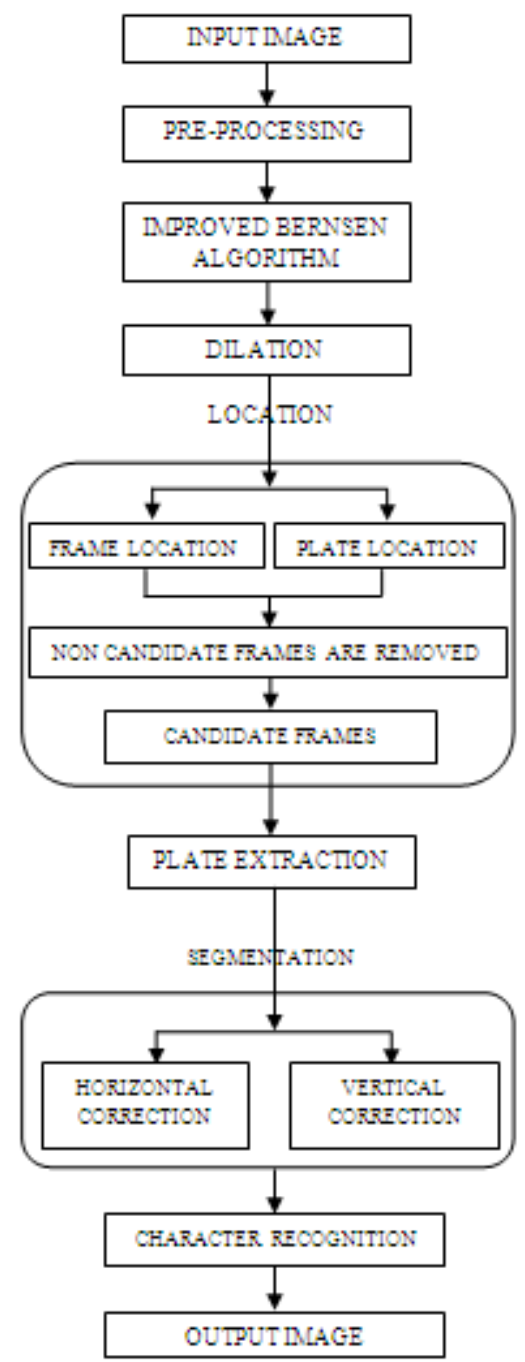

Fig.1.Flowchart of license plate detection algorithm

\section{A. BERNSEN ALGORITHM}

This is the preprocessing step in license plate detection. Bernsen algorithm is proposed for uneven illumination, particularly for shadow removal. Let $\mathrm{f}(\mathrm{x}, \mathrm{y})$ denotes a gray value of point $(\mathrm{x}, \mathrm{y})$.Consider a block whose center is a point $(x, y)$ and size is $(2 \mathrm{w}+1) \times(2 \mathrm{w}+1)$. The threshold $\mathrm{T}(\mathrm{x}, \mathrm{y})$ of $\mathrm{f}(\mathrm{x}, \mathrm{y})$ is computed by

$$
T_{1}(x, y)=\frac{\max _{-w \leq k, l \leq w} f(x+l, y+k)+\min _{-w \leq k, l \leq w} f(x+l, y+k)}{2}
$$

Bernsen algorithm is sensitive to noise, which disturbs the characters' extraction.

Suppose that $\mathrm{f}(\mathrm{x}, \mathrm{y})$ denotes the gray value obtained with the Gaussian filter, $\sigma$ is the scale of the Gaussian filter, and $\mathrm{k}$ and $\mathrm{l}$ are the parameters of the window. An improved Bernsen algorithm is depicted here. Step 1) Compute the threshold $\mathrm{T}_{1}(\mathrm{x}, \mathrm{y})$ of $\mathrm{f}(\mathrm{x}, \mathrm{y})$

$$
T_{1}(x, y)=\frac{\max _{-w \leq k, l \leq w} f(x+l, y+k)+\min _{-w \leq k, l \leq w} f(x+l, y+k)}{2}
$$

Step 2) Create the Gaussian filter for the window $s=(2 w+1) \times(2 w+1)$ of $f(x, y)$. Step 3) Compute the threshold $T_{2}(x, y)$ of $f^{\wedge}(x, y)$ as

$$
T_{2}(x, y)=\frac{\max _{-w \leq k, l \leq w} \hat{f}(x+l, y+k)+\min _{-w \leq k, l \leq w} \hat{f}(x+l, y+k)}{2}
$$

Step 4) To remove noise applying median Filter. 


\section{B. DILATE AN IMAGE OF BERNSEN ALGORITHM}

Dilation is a process of improvising given image by filling holes in an image, sharpen the edges of objects in an image, and join the broken lines and increase the brightness of an image. Using dilation, the noise with-in an image can also be removed. By making the edges sharper, the difference of gray value between neighboring pixels at the edge of an object can be increased. This enhances the edge detection.

In Number Plate Detection, the image of a car plate may not always contain the same brightness and shades. Therefore, the given image has to be converted from RGB to gray form. The dilation operation usually used for probing and expanding the shapes contained in the input image.

\section{LICENSE PLATE LOCATION}

The next step is to locate the license plate. The result of the location is the key for following work. Connected component analysis (CCA) is an important operation in motion detection, which scans an image and labels its pixels into components based on pixel connectivity. Every binary image contains only two labels. ' 0 ' and ' 255 ' which represents the background and foreground pixels respectively. The object is extracted using the connected component labelling operation, which consist of assigning a unique label to each maximally connected foreground region of pixels. License plate detection is of two procedures. License plate location with frame, based on the prior knowledge of the license plate is detected. License plate location without frame, which detects the plate by large numerical extraction.

\section{SEGMENTATION}

The next step is to find all the regions in an image that has high probability of containing a license plate. Co-ordinates of all such probable regions are stored in an array. The output of segmentation process is all the regions that have maximum probability of containing a license plate.

\section{A. HORIZONTAL AND VERTICAL CORRECTION}

In this Number Plate Detection algorithm, the horizontal and vertical histogram, this represents the column-wise and row-wise histogram respectively. These histograms represent the sum of differences of gray values between neighboring pixels of an image, column-wise and row-wise.

In the above step, first the horizontal correction is calculated. To find a horizontal correction, the algorithm traverses through each column of an image. In each column, the algorithm starts with the second pixel from the top. The difference between second and first pixel is calculated. If the difference exceeds certain threshold, it is added to total sum of differences. Then, algorithm will move downwards to calculate the difference between the third and second pixels. So on, it moves until the end of a column and calculate the total sum of differences between neighboring pixels. At the end, an array containing the column-wise sum is created. The same process is carried out to find the vertical correction. In this case, rows are processed instead of columns.

Out of these regions, the one with the maximum histogram value is considered as the most probable candidate for number plate. All the regions are processed row-wise and column-wise to find a common region having maximum horizontal and vertical histogram value. This is the region having highest probability of containing a license plate. This algorithm was verified using several input images having resolution varying from $680 * 480$ to $1600 * 1200$. The images contained vehicles of different colors and varying intensity of light. With all such images, the algorithm correctly recognized the number plate. This algorithm was also tried on images having number plate aligned at certain angle (approximately 8-10 degree) to horizontal axis. Even with such images, the number plates were detected successfully. After successfully implementing and verifying the algorithm in MATLAB, it was coded in $\mathrm{C}$ for implementation on actual hardware.

\section{CHARACTER RECOGNITION}

The most difficult stage in the license plate recognition is character recognition. Direct template matching can be used to identify the characters. Optical character recognition technology is an important technique in character recognition. Train up the different font styles of alphabets and numerical in optical character recognition system. So that the license plate with different font styles can easily recognize. After recognize the number plate the final output is displayed in notepad.

\section{EXPERIMENTAL RESULTS}

In the paper was tested with 100 images, such as an Indian vehicle images using different backgrounds. The vertical correction algorithm can efficiently separate each character of whole license plate. Bernsen algorithm proposed in this paper just can be used in black character in the white background. 


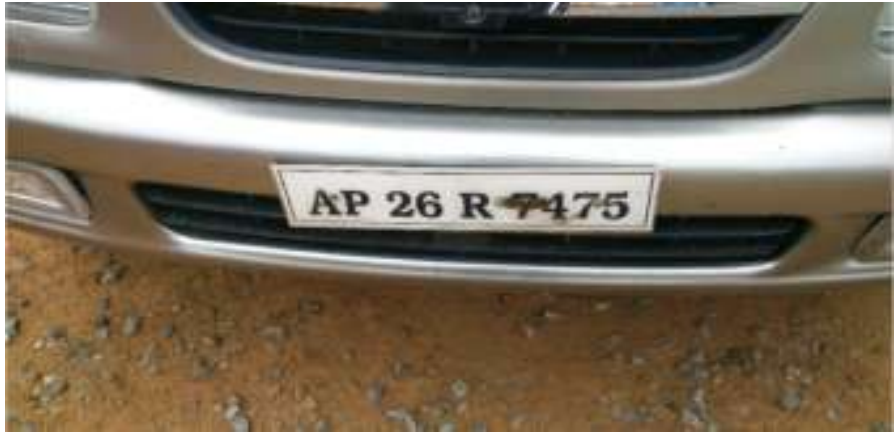

Fig.2. Original Image

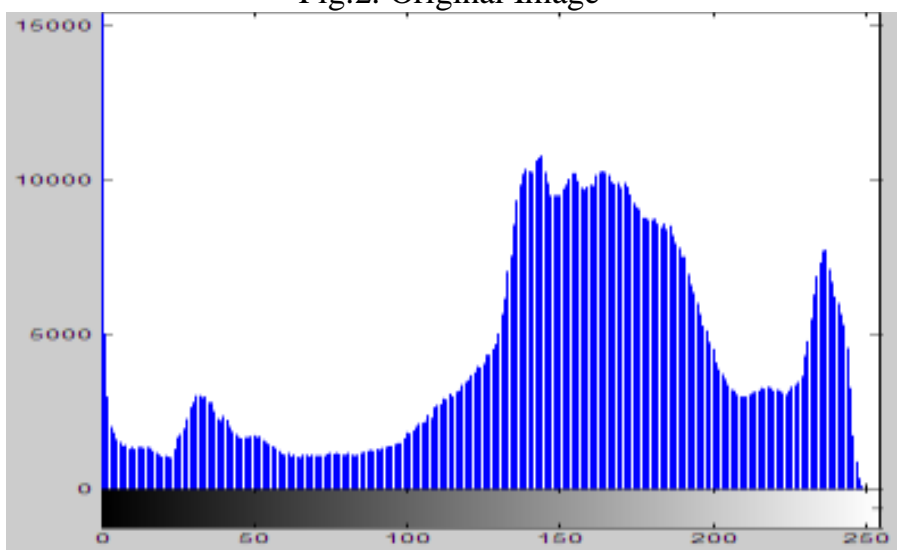

Fig.3. Histogram

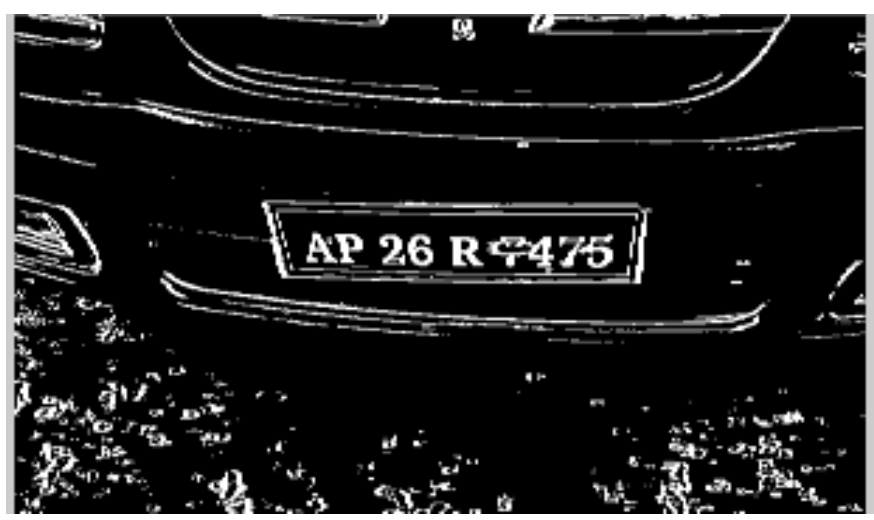

Fig.4. Improved Bernsen Algorithm

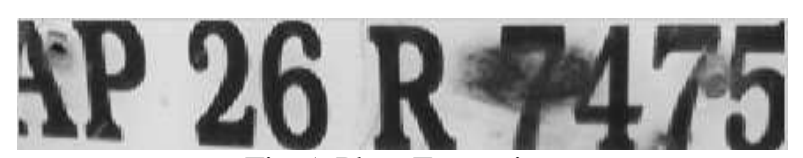

Fig.5. Plate Extraction

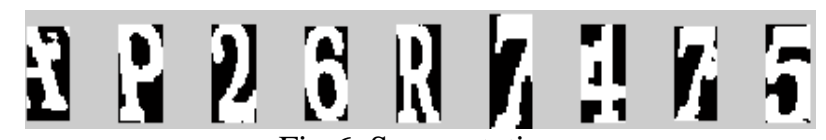

Fig.6. Segmentation 


text - Notepad
File Edit Format View
AP26R7475

Fig.7. Character Recognition

VI. CONCLUSION

The work focuses on a solution for image disturbance resulting for uneven illumination and various outdoor conditions such as shadow and exposure while generally difficult for obtaining successful processed results are using traditional methods and Bernsen algorithm. The proposed method can divide the characters of plate effectively based on the segmentation methods. Experimental results show the proposed method. The proposed algorithm is an effective method to recognize the Indian license plate. It could be improved for recognizing all countries license plates. Further improvement is made possible by improving the segmentation methods which is also possible to segment the double row number plate.

\section{References}

[1] Ying Wen, Yue Lu, IEEE, Jingqi Yan, Zhenyu Zhou, Karen M. von Deneen, and Pengfei Shi, “ An Algorithm for License Plate Recognition Applied to Intelligent Transportation System” IEEE TRANSACTIONS ON INTELLIGENT TRANSPORTATION SYSTEMS, VOL. 12, NO. 3, SEPTEMBER 2011

[2] Jing-Ming Guo and Yun-Fu Liu "License Plate location and character segmentation with Feedback self learning and Hybrid Binarization techniques", IEEE TRANSACTIONS ON VEHICULAR TECHNOLOGY, VOL. 57, NO. 3, MAY 2008

[3] Q. Zuo and Z. Shi, "A real-time algorithm for license plate extraction based on mathematical morphology," J. Image Graph., vol. 8, no. 3, pp 281-285, 2003

[4] Halina Kwasnicka and Bartosz Wawrzyniak, "License plate localization and recognition in camera pictures", AI-METH 2002, November 13-15, 2002.

[5] Y. P. Huang, C. H. Chen, Y. T. Chang, and F. E Sandnes, "An intelligent strategy for checking the annual inspection status of motorcycles based on license plate recognition," Expert Syst. Appl., vol. 36, no. 5, pp. 9260-9267, Jul. 2009.

[6] D. N. Zheng, Y. N. Zhao, and J. X. Wang, “An efficient method of license plate location," Pattern Recognit. Lett., vol. 26, no. 15, pp. 2431-2438, Nov. 2005.

[7] J. B. Jiao, Q. X. Ye, and Q. M. Huang, “A configurable method for multi-style license plate recognition,” Pattern Recognit., vol. 42, no. 3, pp. 358-369, Mar. 2009.

[8] V. Abolghasemi and A. Ahmadyfard, “An edge based color-aided methodfor license plate detection,” Image Vis. Comput., vol. 27, no. 8, pp. 1134-1142, Jul. 2009.

[9] S. Luis, M. Jose, R. Enrique, and G. Narucuso, "Automatic car plate detection and recognition through intelligent vision engineering," in Proc.IEEE 33rd Int. Carnahan Conf. Security Technol., 1999, pp. 71-76.

[10] N. Otsu, "A threshold selection method from gray-level histograms," IEEE Trans. Syst., Man, Cybern., vol. SMC-9, no. 1, pp. 6266, Jan. 1979. 